\title{
SINGULAR FRACTIONAL CONTINUOUS-TIME AND DISCRETE-TIME LINEAR SYSTEMS
}

\author{
Tadeusz KACZOREK* \\ *Faculty of Electrical Engineering, Bialystok University of Technology, ul. Wiejska 45D, 15-351 Białystok, Poland \\ kaczorek@isep.pw.edu.pl
}

\begin{abstract}
New classes of singular fractional continuous-time and discrete-time linear systems are introduced. Electrical circuits are example of singular fractional continuous-time systems. Using the Caputo definition of the fractional derivative, the Weierstrass regular pencil decomposition and Laplace transformation the solution to the state equation of singular fractional linear systems is derived. It is shown that every electrical circuit is a singular fractional systems if it contains at least one mesh consisting of branches with only ideal supercondensators and voltage sources or at least one node with branches with supercoils. Using the Weierstrass regular pencil decomposition the solution to the state equation of singular fractional discrete-time linear systems is derived. The considerations are illustrated by numerical examples.
\end{abstract}

Keywords: Fractional, Singular, Linear Circuit, Regular Pencil, Solution, Supercondensator, Supercoil

\section{INTRODUCTION}

Singular (descriptor) linear systems have been addressed in many papers and books (Benvenuti and Farina, 2004; Dodog and Stosic, 2009; Dail, 1989; Kaczorek, 1992, 2004, 2008, 2010, 2011; Podlubny, 1999). The eigenvalues and invariants assignment by state and output feedbacks have been investigated in Benvenuti and Farina (2004), Dodog and Stosic (2009), Dail, (1989), Kaczorek $(2004,2008)$ and the realization problem for singular positive continuous-time systems with delays in Kaczorek (2010). The computation of Kronecker's canonical form of a singular pencil has been analyzed in Podlubny (1999).

Fractional positive continuous-time linear systems have been addressed in Kaczorek (2010) and positive linear systems with different fractional orders in Kaczorek (2007). An analysis of fractional linear electrical circuits has been presented in Gantmacher (1960) and some selected problems in theory of fractional linear systems in the monograph Kaczorek (2007).

In this paper a new class of singular fractional linear systems and electrical circuits will be introduced and their solution of state equations will be derived.

The paper is organized as follows. In section 2 the Caputo definition of the fractional derivative and the solution to the state equation of the fractional linear system are recalled. The solution of the state equation of singular fractional linear system is derived using the Weierstrass pencil decomposition and the Laplace transform in Section 3. Singular fractional linear electrical circuits are introduced in Section 4 . In section 5 the fractional singular discrete-time linear systems are introduced and Weierstrass regular pencil decomposition is recalled. The solution of the state equation of singular fractional linear discrete-time system is derived using the Weierstrass pencil decomposition in Section 6 . Illustrating numerical examples are given in Section 7. Concluding remarks are given in Section 8.

To the best of the author's knowledge singular fractional linear systems and electrical circuits have not been considered yet.
The following notation will be used in the paper.

The set of $n \times m$ real matrices will be denoted by $\mathfrak{R}^{n \times m}$ and $\mathfrak{R}^{n}:=\mathfrak{R}^{n \times 1}$. The set of $m \times n$ real matrices with nonnegative entries will be denoted by $\mathfrak{R}_{+}^{m \times n}$ and $\mathfrak{R}_{+}^{n}:=\mathfrak{R}_{+}^{n \times 1}$. The set of nonnegative integers will be denoted by $Z_{+}$and the $n \times n$ identity matrix by $I_{n}$.

\section{FRACTIONAL CONTINUOUS-TIME LINEAR SYSTEMS}

The following Caputo definition of the fractional derivative will be used (Kaczorek, 2007; Kucera and Zagalak, 1988):

$$
\begin{aligned}
& \frac{d^{\alpha}}{d t^{\alpha}} f(t)=\frac{1}{\Gamma(n-\alpha)} \int_{0}^{t} \frac{f^{(n)}}{(t-\tau)^{\alpha+1-n}} d \tau, \\
& n-1<\alpha \leq n \in N=\{1,2, \ldots\}
\end{aligned}
$$

where $\alpha \in \Re$ is the order of fractional derivative, $f^{n}(\tau)=\frac{d^{n} f(\tau)}{d \tau^{n}}$ and $\Gamma(x)=\int_{0}^{\infty} e^{-t} t^{x-1} d t$ is the gamma function.

Consider the continuous-time fractional linear system described by the state equation:

$$
\frac{d^{\alpha}}{d t^{\alpha}} x(t)=A x(t)+B u(t), \quad 0<\alpha \leq 1
$$

where $x(t) \in \Re^{n}, u(t) \in \Re^{m}$ are the state and input vectors and $A \in \Re^{n \times n}, B \in \Re^{n \times m}$.

Theorem 2.1. The solution of equation (2.2) is given by:

$$
x(t)=\Phi_{0}(t) x_{0}+\int_{0}^{t} \Phi(t-\tau) B u(\tau) d \tau, \quad x(0)=x_{0}
$$

where: 


$$
\begin{aligned}
& \Phi_{0}(t)=\sum_{k=0}^{\infty} \frac{A^{k} t^{k \alpha}}{\Gamma(k \alpha+1)} \\
& \Phi(t)=\sum_{k=0}^{\infty} \frac{A^{k} t^{(k+1) \alpha-1}}{\Gamma[(k+1) \alpha]}
\end{aligned}
$$

Proof is given in Kaczorek (2010a, b).

Remark 2.1. From (2.4) and (2.5) for $\alpha=1$ we have:

$\Phi_{0}(t)=\Phi(t)=\sum_{k=0}^{\infty} \frac{(A t)^{k}}{\Gamma(k+1)}=e^{A t}$.

\section{SINGULAR FRACTIONAL CONTINUOUS-TIME LINEAR SYSTEMS}

Consider singular fractional linear system described by the state equations:

$$
\begin{aligned}
& E \frac{d^{\alpha}}{d t^{\alpha}} x(t)=A x(t)+B u(t) \\
& y(t)=C x(t)+D u(t)
\end{aligned}
$$

where $x(t) \in \mathfrak{R}^{n}, u(t) \in \mathfrak{R}^{m}, y(t) \in \mathfrak{R}^{p}$ are the state, input and output vectors and $E, A \in \mathfrak{R}^{n \times n}, B \in \mathfrak{R}^{n \times m}, C \in \mathfrak{R}^{p \times n}$, $D \in \mathfrak{R}^{p \times m}$.

The initial condition for (3.1a) is given by:

$$
x(0)=x_{0}
$$

It is assumed that the pencil of the pair $(E, A)$ is regular, i.e.

$$
\operatorname{det}[E s-A] \neq 0
$$

for some $z \in C$ (the field of complex numbers). It is well-known (Fahmy and O'reill, 1989; Kaczorek, 1992)) that if the pencil is regular then there exists a pair of nonsingular matrices $P, Q \in$ $\mathfrak{R}^{n \times n}$ such that:

$$
P[E s-A] Q=\left[\begin{array}{cc}
I_{n_{1}} & 0 \\
0 & N
\end{array}\right] s-\left[\begin{array}{cc}
A_{1} & 0 \\
0 & I_{n_{2}}
\end{array}\right]
$$

where: $n_{1}$ is equal to degree of the polynomial $\operatorname{det}[E s-A]$, $A_{1} \in \mathfrak{R}^{n_{1} \times n_{1}}, N \in \mathfrak{R}^{n_{2} \times n_{2}}$ is a nilpotent matrix with the index $\mu$ (i.e. $N^{\mu}=0$ and $N^{\mu-1} \neq 0$ and $n_{1}+n_{2}=n$ ).

Applying to the Eq. (3.1a) with zero initial conditions $x_{0}=0$ the Laplace transform $(\mathcal{L})$ we obtain:

$\left[E s^{\alpha}-A\right] X(s)=B U(s)$

where $\mathrm{X}(\mathrm{s})=\mathcal{L}[\mathrm{x}(\mathrm{t})]=\int_{0}^{\infty} \mathrm{x}(\mathrm{t}) \mathrm{e}^{-\mathrm{st}} \mathrm{dt}$ and $\mathrm{U}(\mathrm{s})=\mathcal{L}[\mathrm{u}(\mathrm{t})]$. By assumption (3.2) the pencil $\left[\mathrm{Es}^{\alpha}-\mathrm{A}\right]$ is regular and we may apply the decomposition (3.3) to Eq. (3.1a).

Premultiplying Eq. (3.1a) by the matrix $P \in \mathfrak{R}^{n \times n}$ and introducing the new state vector:

$$
\bar{x}(t)=Q^{-1} x(t)=\left[\begin{array}{l}
x_{1}(t) \\
x_{2}(t)
\end{array}\right], x_{1}(t) \in \mathfrak{R}^{n_{1}}, x_{2}(t) \in \mathfrak{R}^{n_{2}}
$$

$$
\begin{aligned}
& \frac{d^{\alpha}}{d t^{\alpha}} x_{1}(t)=A_{1} x_{1}(t)+B_{1} u(t) \\
& N \frac{d^{\alpha}}{d t^{\alpha}} x_{2}(t)=x_{2}(t)+B_{2} u(t)
\end{aligned}
$$

where:

$$
P B=\left[\begin{array}{c}
B_{1} \\
B_{2}
\end{array}\right], B_{1} \in \mathfrak{R}^{n_{1} \times m}, B_{2} \in \mathfrak{R}^{n_{2} \times m}
$$

Using (2.3) we obtain the solution to Eq. (3.6a) in the form:

$$
x_{1}(t)=\Phi_{10}(t) x_{10}+\int_{0}^{t} \Phi_{11}(t-\tau) B_{1} u(\tau) d \tau
$$

where:

$$
\begin{aligned}
& \Phi_{10}(t)=\sum_{k=0}^{\infty} \frac{A_{1}{ }^{k} t^{k \alpha}}{\Gamma(k \alpha+1)} \\
& \Phi_{11}(t)=\sum_{k=0}^{\infty} \frac{A_{1}{ }^{k} t^{(k+1) \alpha-1}}{\Gamma[(k+1) \alpha]}
\end{aligned}
$$

and $x_{10} \in \mathfrak{R}^{n_{1}}$ is the initial condition for (3.6a) defined by:

$\left[\begin{array}{l}x_{10} \\ x_{20}\end{array}\right]=Q^{-1} x_{0}, x_{0}=x(0)$

To find the solution of Eq. (3.6b) we apply to the equation the Laplace transform and we obtain:

$$
N s^{\alpha} X_{2}(s)-N s^{\alpha-1} x_{20}=X_{2}(s)+B_{2} U(s)
$$

Since Kaczorek (2010a) and (Dodog and Stosic, 2009) for $0<\alpha<1$

$$
\mathcal{L}\left[\frac{d^{\alpha}}{d t^{\alpha}} x_{2}(t)\right]=s^{\alpha} X_{2}(s)-s^{\alpha-1} x_{20}
$$

where $X_{2}(s)=\mathcal{L}\left[x_{2}(t)\right]$. From (3.8) we have:

$$
X_{2}(s)=\left[N s^{\alpha}-I_{n_{2}}\right]^{-1}\left(B_{2} U(s)+N s^{\alpha-1} x_{20}\right)
$$

It is easy to check that:

$$
\left[N s^{\alpha}-I_{n_{2}}\right]^{-1}=-\sum_{i=0}^{\mu-1} N^{i} s^{i \alpha}
$$

since:

$\left[N s^{\alpha}-I_{n_{2}}\right]\left(-\sum_{i=0}^{\mu-1} N^{i} s^{i \alpha}\right)=I_{n_{2}}$

and $N^{i}=0$ for $i=\mu, \mu+1, \ldots$

Substitution of (3.10) into (3.9) yields:

we obtain: 


$$
\begin{aligned}
& X_{2}(s)=-B_{2} U(s)-\frac{N x_{20}}{s^{1-\alpha}} \\
& -\sum_{i=1}^{\mu-1}\left[N^{i} B_{2} s^{i \alpha} U(s)+N^{i+1} s^{(i+1) \alpha-1} x_{20}\right]
\end{aligned}
$$

Using inverse Laplace transform $\left(\mathcal{L}^{-1}\right)$ to $(3.12)$ and the convolution theorem we obtain for $1-\alpha>0$ :

$$
\begin{gathered}
x_{2}(t)=\mathcal{L}^{-1}\left[X_{2}(s)\right]=-B_{2} u(t)-N x_{20} \frac{t^{-\alpha}}{\Gamma(1-\alpha)} \\
-\sum_{i=1}^{\mu-1}\left[N^{i} B_{2} \frac{d^{i \alpha}}{d t^{i \alpha}} u(t)+N^{i+1} \frac{d^{(i+1) \alpha-1}}{d t^{(i+1) \alpha-1}} x_{20}\right]
\end{gathered}
$$

since $\mathcal{L}^{-1}\left[\frac{1}{s^{\alpha+1}}\right]=\frac{t^{\alpha}}{\Gamma(1+\alpha)}$ for $\alpha+1>0$.

Therefore the following theorem has been proved.

Theorem 3.1. The solution to Eq. (3.1a) with the initial condition (3.1c) has the form

$$
x(t)=Q\left[\begin{array}{l}
x_{1}(t) \\
x_{2}(t)
\end{array}\right]
$$

where $x_{1}(t)$ and $x_{2}(t)$ are given by (3.7) and (3.13) respectively. Knowing the solution (3.14) we can find the output $y(t)$ of the system using the formula

$$
y(t)=C Q\left[\begin{array}{l}
x_{1}(t) \\
x_{2}(t)
\end{array}\right]+D u(t)
$$

\section{SINGULAR FRACTIONAL ELECTRICAL CIRCUITS}

Let the current $i_{C}(t)$ in the supercondensator with the capacity $C$ be the $\alpha$ order derivative of its charge $q(t)$ (Gantmacher, 1960):

$i_{C}(t)=\frac{d^{\alpha} q(t)}{d t^{\alpha}}$

Taking into account that $q(t)=C u_{C}(t)$ we obtain:

$i_{C}(t)=C \frac{d^{\alpha} u_{C}(t)}{d t^{\alpha}}$

where $u_{C}(t)$ is the voltage on the supercondensator.

Similarly, let the voltage $u_{L}(t)$ on the supercoil (inductor) with the inductance $L$ be the $\beta$ order derivative of its magnetic flux $\psi(t)$ :

$u_{L}(t)=\frac{d^{\beta} \Psi(t)}{d t^{\beta}}$

Taking into account that $\psi(t)=L i_{L}(t)$ we obtain

$u_{L}(t)=L \frac{d^{\beta} i_{L}(t)}{d t^{\beta}}$

where $i_{L}(t)$ is the current in the supercoil.
Example 4.1. Consider electrical circuit shown in Fig. 1 with given resistance $R$, capacitances $C_{1}, C_{2}, C_{3}$ and source voltages $e_{1}$ and $e_{2}$.

Using the Kirchhoff's laws we can write for the electrical circuit the equations:

$e_{1}=R C_{1} \frac{d^{\alpha} u_{1}}{d t^{\alpha}}+u_{1}+u_{3}$

$C_{1} \frac{d^{\alpha} u_{1}}{d t^{\alpha}}+C_{2} \frac{d^{\alpha} u_{2}}{d t^{\alpha}}-C_{3} \frac{d^{\alpha} u_{3}}{d t^{\alpha}}=0$

$e_{2}=u_{2}+u_{3}$

The equations (4.5) can be written in the form:

$$
\begin{aligned}
& {\left[\begin{array}{ccc}
R C_{1} & 0 & 0 \\
C_{1} & C_{2} & -C_{3} \\
0 & 0 & 0
\end{array}\right] \frac{d^{\alpha}}{d t^{\alpha}}\left[\begin{array}{l}
u_{1} \\
u_{2} \\
u_{3}
\end{array}\right]=\left[\begin{array}{ccc}
-1 & 0 & -1 \\
0 & 0 & 0 \\
0 & -1 & -1
\end{array}\right]\left[\begin{array}{l}
u_{1} \\
u_{2} \\
u_{3}
\end{array}\right]} \\
& +\left[\begin{array}{ll}
1 & 0 \\
0 & 0 \\
0 & 1
\end{array}\right]\left[\begin{array}{l}
e_{1} \\
e_{2}
\end{array}\right]
\end{aligned}
$$

In this case we have:

$E=\left[\begin{array}{ccc}R C_{1} & 0 & 0 \\ C_{1} & C_{2} & -C_{3} \\ 0 & 0 & 0\end{array}\right], \quad A=\left[\begin{array}{ccc}-1 & 0 & -1 \\ 0 & 0 & 0 \\ 0 & -1 & -1\end{array}\right], \quad B=\left[\begin{array}{ll}1 & 0 \\ 0 & 0 \\ 0 & 1\end{array}\right]$

Note that the matrix $E$ is singular (det $E=0)$ but the pencil:

$$
\begin{gathered}
\operatorname{det}\left[E s^{\alpha}-A\right]=\left|\begin{array}{ccc}
R C_{1} s^{\alpha}+1 & 0 & 1 \\
C_{1} s^{\alpha} & C_{2} s^{\alpha} & -C_{3} s^{\alpha} \\
0 & 1 & 1
\end{array}\right| \\
=\left(R C_{1} s^{\alpha}+1\right)\left(C_{2}+C_{3}\right) s^{\alpha}+C_{1} s^{\alpha}
\end{gathered}
$$

is regular. Therefore, the electrical circuit is a singular fractional linear system.

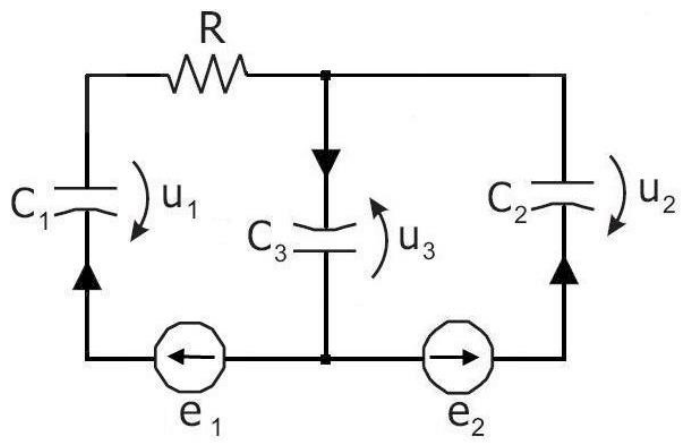

Fig.1. Electrical circuit

Remark 4.1. If the electrical circuit contains at least one mesh consisting of branches with only ideal supercondensators and voltage sources then its matrix $E$ is singular since the row corresponding to this mesh is zero row. This follows from the fact that the equation written by the use of the voltage Kirchhoff's law is algebraic one. 
Example 4.2. Consider electrical circuit shown in Fig. 2 with given resistances $R_{1}, R_{2}, R_{3}$ inductances $L_{1}, L_{2}, L_{3}$ and source voltages $e_{1}$ and $e_{2}$.

Using the Kirchhoff's laws we can write for the electrical circuit the equations:

$$
\begin{aligned}
& e_{1}=R_{1} i_{1}+L_{1} \frac{d^{\beta} i_{1}}{d t^{\beta}}+R_{3} i_{3}+L_{3} \frac{d^{\beta} i_{3}}{d t^{\beta}} \\
& e_{2}=R_{2} i_{2}+L_{2} \frac{d^{\beta} i_{2}}{d t^{\beta}}+R_{3} i_{3}+L_{3} \frac{d^{\beta} i_{3}}{d t^{\beta}} \\
& i_{1}+i_{2}-i_{3}=0
\end{aligned}
$$

The equations (4.9) can be written in the form:

$$
\begin{aligned}
& {\left[\begin{array}{ccc}
L_{1} & 0 & L_{3} \\
0 & L_{2} & L_{3} \\
0 & 0 & 0
\end{array}\right] \frac{d^{\beta}}{d t^{\beta}}\left[\begin{array}{l}
i_{1} \\
i_{2} \\
i_{3}
\end{array}\right]=\left[\begin{array}{ccc}
-R_{1} & 0 & -R_{3} \\
0 & -R_{2} & -R_{3} \\
1 & 1 & -1
\end{array}\right]\left[\begin{array}{l}
i_{1} \\
i_{2} \\
i_{3}
\end{array}\right]} \\
& +\left[\begin{array}{ll}
1 & 0 \\
0 & 1 \\
0 & 0
\end{array}\right]\left[\begin{array}{l}
e_{1} \\
e_{2}
\end{array}\right]
\end{aligned}
$$

In this case we have:

$E=\left[\begin{array}{ccc}L_{1} & 0 & L_{3} \\ 0 & L_{2} & L_{3} \\ 0 & 0 & 0\end{array}\right], A=\left[\begin{array}{ccc}-R_{1} & 0 & -R_{3} \\ 0 & -R_{2} & -R_{3} \\ 1 & 1 & -1\end{array}\right], B=\left[\begin{array}{ll}1 & 0 \\ 0 & 1 \\ 0 & 0\end{array}\right]$ (4.11)

Note that the matrix $E$ is singular but the pencil:

$$
\begin{aligned}
& \operatorname{det}\left[E s^{\beta}-A\right]=\left|\begin{array}{ccc}
L_{1} s^{\beta}+R_{1} & 0 & L_{3} s^{\beta}+R_{3} \\
0 & L_{2} s^{\beta}+R_{2} & L_{3} s^{\beta}+R_{3} \\
-1 & -1 & 1
\end{array}\right| \\
& \quad=\left[L_{1}\left(L_{2}+L_{3}\right)+L_{2} L_{3}\right] s^{2 \beta} \\
& \quad+\left[\left(L_{2}+L_{3}\right) R_{1}+\left(L_{1}+L_{3}\right) R_{2}+\left(L_{1}+L_{2}\right) R_{3}\right] s^{\beta} \\
& \quad+R_{1}\left(R_{2}+R_{3}\right)+R_{2} R_{3}
\end{aligned}
$$

is regular. Therefore, the electrical circuit is a singular fractional linear system.

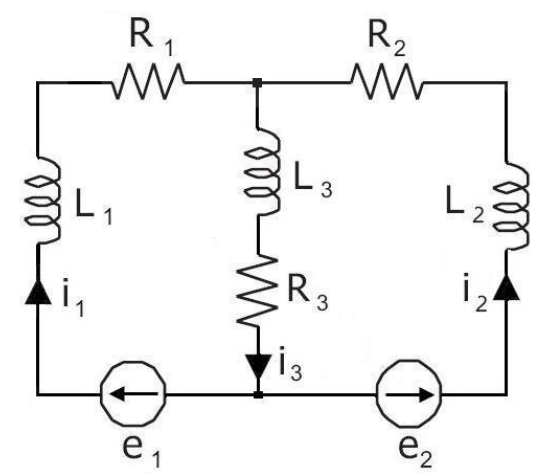

Fig.2. Electrical circuit

Remark 4.2. If the electrical circuit contains at least one node with branches with supercoils then its matrix $E$ is singular since it has at least one zero row. This follows from the fact that the equation written using the current Kirchhoff's law for this node is algebraic one.

In general case we have the following theorem.

Theorem 4.1. Every electrical circuit is a singular fractional system if it contains at least one mesh consisting of branches with only ideal supercondensators and voltage source or at least one node with branches with supercoils.

Proof. By Remark 2.1 the matrix $E$ of the system is singular if the electrical circuit contains at least one mesh consisting of branches with only ideal supercondensators and voltage source. Similarly by Remark 2.2 the matrix $E$ is singular if the electrical circuit contains at least one node with branches with supercoils.

Using the solution (3.14) of Eq. (3.1a) we may find the voltages on the supercondensators and currents in the supercoils in the transient states of the singular fractional linear electrical circuits. Knowing the voltages and currents and using (3.15) we may find also any currents and voltages in the singular fractional linear electrical circuits.

Example 4.3. (an continuation of Example 4.1)

Using one of the well-known methods (Podlubny, 1999; Dodig and Stosic, 2009; Kaczorek, 1992) we can find for the pencil (4.8) the matrices:

$$
P=\left[\begin{array}{ccc}
\frac{1}{R C_{1}} & 0 & -\frac{C_{2}}{R C_{1}\left(C_{2}+C_{3}\right)} \\
-\frac{1}{R\left(C_{2}+C_{3}\right)} & \frac{1}{C_{2}+C_{3}} & \frac{C_{2}}{R\left(C_{2}+C_{3}\right)^{2}} \\
0 & 0 & -1
\end{array}\right],
$$

$$
Q=\left[\begin{array}{ccc}
1 & 0 & 0 \\
0 & 1 & \frac{C_{3}}{C_{2}+C_{3}} \\
0 & -1 & \frac{C_{2}}{C_{2}+C_{3}}
\end{array}\right]
$$

which transform it to the canonical form (3.3) with:

$$
\begin{aligned}
& A_{1}=\left[\begin{array}{cc}
-\frac{1}{R C_{1}} & \frac{1}{R C_{1}} \\
\frac{1}{R\left(C_{2}+C_{3}\right)} & -\frac{1}{R\left(C_{2}+C_{3}\right)}
\end{array}\right], \\
& N=[0], \quad n_{1}=2, \quad n_{2}=1
\end{aligned}
$$

Using the matrix $B$ given by (4.7), (4.13) and (3.6c) we obtain:

$$
\left[\begin{array}{c}
B_{1} \\
B_{2}
\end{array}\right]=P B=\left[\begin{array}{cc}
\frac{1}{R C_{1}} & -\frac{C_{2}}{R C_{1}\left(C_{2}+C_{3}\right)} \\
-\frac{1}{R\left(C_{2}+C_{3}\right)} & \frac{C_{2}}{R\left(C_{2}+C_{3}\right)^{2}} \\
0 & -1
\end{array}\right]
$$

from (3.7) we have: 


$$
x_{1}(t)=\Phi_{10}(t) x_{10}+\int_{0}^{t} \Phi_{11}(t-\tau) B_{1} u(\tau) d \tau
$$

for any given initial condition $x_{10} \in \mathfrak{R}^{n_{1}}$ and input $u(t)$, where:

$$
\Phi_{10}(t)=\sum_{k=0}^{\infty} \frac{A_{1}{ }^{k} t^{k \alpha}}{\Gamma(k \alpha+1)}, \Phi_{11}(t)=\sum_{k=0}^{\infty} \frac{A_{1}{ }^{k} t^{(k+1) \alpha-1}}{\Gamma[(k+1) \alpha]},
$$$$
0<\alpha<1
$$

In this case using (3.13) we obtain:

$$
x_{2}(t)=-B_{2} u(t)
$$

since $N=[0]$.

In a similar way we may find currents in the supercoils of the singular fractional electrical circuit shown in Fig. 2.

\section{FRACTIONAL DISCRETE-TIME LINEAR SYSTEMS}

Consider the singular fractional discrete-time linear system described by the state equation:

$$
E \Delta^{\alpha} x_{i+1}=A x_{i}+B u_{i}, i \in Z_{+}=\{0,1, \ldots\}
$$

where: $x_{i} \in \mathfrak{R}^{n}, u_{i} \in \mathfrak{R}^{m}$ are the state and input vectors, $A \in \mathfrak{R}^{n \times n}, E \in \mathfrak{R}^{n \times n}, B \in \mathfrak{R}^{n \times m}$ and the fractional difference of the order $\alpha$ is defined by:

$$
\begin{aligned}
& \Delta^{\alpha} x_{i}=\sum_{k=0}^{i}(-1)^{k}\left(\begin{array}{l}
\alpha \\
k
\end{array}\right) x_{i-k}, 0<\alpha<1 \\
& \left(\begin{array}{l}
\alpha \\
k
\end{array}\right)=\left\{\begin{array}{cc}
1 & \text { for } k=0 \\
\frac{\alpha(\alpha-1) \ldots(\alpha-k+1)}{k !} & \text { for } k=1,2, \ldots
\end{array}\right.
\end{aligned}
$$

It is assumed that:

$$
\operatorname{det} E=0
$$

and:

$$
\operatorname{det}[E z-A] \neq 0
$$

for some $z \in C$ (the field of complex numbers).

Lemma 5.1. (Fahmy and O'Reill, 1989; Kaczorek, 1992) If (5.4) holds then there exist nonsingular matrices $P, Q \in \mathfrak{R}^{n \times n}$ such that:

$$
P E Q=\left[\begin{array}{cc}
I_{n_{1}} & 0 \\
0 & N
\end{array}\right], \quad P A Q=\left[\begin{array}{cc}
A_{1} & 0 \\
0 & I_{n_{2}}
\end{array}\right]
$$

where: $N \in \mathfrak{R}^{n_{2} \times n_{2}}$ is a nilpotent matrix with the index $\mu$ (i.e. $N^{\mu}=0$ and $\left.N^{\mu-1} \neq 0\right), A_{1} \in \Re^{n_{1} \times n_{1}}, n_{1}$ is equal to degree of the polynomial:

$$
\operatorname{det}[E s-A]=a_{n_{1}} z^{n_{1}}+\ldots+a_{1} z+a_{0}
$$

and $n_{1}+n_{2}=n$.

A method for computation of the matrices $P$ and $Q$ has been given in Van Dooren (1979).
Using Lemma 5.1 we shall derive the solution $x_{i}$ to the equation (5.1) for a given initial conditions $x_{0}$ and an input vector $u_{i}, i \in Z_{+}$.

\section{SOLUTION OF THE SINGULAR FRACTIONAL DISCRETE-TIME LINEAR SYSTEMS}

Premultiplying the equation (5.1) by the matrix $P \in \mathfrak{R}^{n \times n}$ and introducing the new state vector:

$\bar{x}_{i}=\left[\begin{array}{c}\bar{x}_{i}^{(1)} \\ \bar{x}_{i}^{(2)}\end{array}\right]=Q^{-1} x_{i}, \bar{x}_{i}^{(1)} \in \mathfrak{R}^{n_{1}}, \bar{x}_{i}^{(2)} \in \mathfrak{R}^{n_{2}}, i \in Z_{+}$

we obtain:

$P E Q Q^{-1} \Delta^{\alpha} x_{i+1}=P E Q \Delta^{\alpha} Q^{-1} x_{i+1}=P A Q Q^{-1} x_{i}+P B u_{i}$ (6.2) and after using (5.5) and (6.1):

$\left[\begin{array}{cc}I_{n_{1}} & 0 \\ 0 & N\end{array}\right] \Delta^{\alpha}\left[\begin{array}{c}\bar{x}_{i+1}^{(1)} \\ \bar{x}_{i+1}^{(2)}\end{array}\right]=\left[\begin{array}{cc}A_{1} & 0 \\ 0 & I_{n_{2}}\end{array}\right]\left[\begin{array}{c}\bar{x}_{i}^{(1)} \\ \bar{x}_{i}^{(2)}\end{array}\right]+\left[\begin{array}{c}B_{1} \\ B_{2}\end{array}\right] u_{i}, i \in Z_{+}$

where:

$\left[\begin{array}{l}B_{1} \\ B_{2}\end{array}\right]=P B, B_{1} \in \mathfrak{R}^{n_{1} \times m}, B_{2} \in \mathfrak{R}^{n_{2} \times m}$

Taking into account (5.2) from (6.3) we obtain:

$$
\begin{gathered}
\bar{x}_{i+1}^{(1)}=-\sum_{k=1}^{i+1}(-1)^{k}\left(\begin{array}{l}
\alpha \\
k
\end{array}\right) \bar{x}_{i-k+1}^{(1)}+A_{1} \bar{x}_{i}^{(1)}+B_{1} u_{i} \\
=A_{1 \alpha} \bar{x}_{i}^{(1)}+\sum_{k=2}^{i+1}(-1)^{k-1}\left(\begin{array}{l}
\alpha \\
k
\end{array}\right) \bar{x}_{i-k+1}^{(1)}+B_{1} u_{i}
\end{gathered}
$$

and:

$N\left[\bar{x}_{i+1}^{(2)}+\sum_{k=1}^{i+1}(-1)^{k}\left(\begin{array}{l}\alpha \\ k\end{array}\right) \bar{x}_{i-k+1}^{(2)}\right]=\bar{x}_{i}^{(2)}+B_{2} u_{i}$

where: $A_{1 \alpha}=A_{1}+I_{n_{1}} \alpha$.

The solution $\bar{x}_{i}^{(1)}$ to the equation (6.5) is well-known (Kaczorek, 2007b; 2010) and it is given by the theorem.

Theorem 6.1. The solution $\bar{x}_{i}^{(1)}$ of the equation (6.5) is given by the formula:

$\bar{x}_{i}^{(1)}=\Phi_{i} \bar{x}_{0}^{(1)}+\sum_{k=0}^{i-1} \Phi_{i-k-1} B_{1} u_{k}, i \in Z_{+}$

where the matrices $\Phi_{i}$ are determined by the equation:

$\Phi_{i+1}=\Phi_{i} A_{1 \alpha}+\sum_{k=2}^{i+1}(-1)^{k-1}\left(\begin{array}{l}\alpha \\ k\end{array}\right) \Phi_{i-k+1} \quad \Phi_{0}=I_{n_{1}}$

To find the solution $\bar{x}_{i}^{(2)}$ of the equation (6.6) for $N \neq 0$ it is assumed that: 


$$
N=\left[\begin{array}{ccccc}
0 & 0 & \ldots & 0 & 0 \\
1 & 0 & \ldots & 0 & 0 \\
0 & 1 & \ldots & 0 & 0 \\
\vdots & \vdots & \ddots & \vdots & \vdots \\
0 & 0 & \ldots & 1 & 0
\end{array}\right] \in \Re^{n_{2}}
$$

For (6.9) the equation (6.6) can be written in the form:

$$
\begin{aligned}
& {\left[\begin{array}{ccccc}
0 & 0 & \ldots & 0 & 0 \\
1 & 0 & \ldots & 0 & 0 \\
0 & 1 & \ldots & 0 & 0 \\
\vdots & \vdots & \ddots & \vdots & \vdots \\
0 & 0 & \ldots & 1 & 0
\end{array}\right]\left(\sum_{j=0}^{i+1}(-1)^{j}\left(\begin{array}{c}
\alpha \\
j
\end{array}\right)\left[\begin{array}{c}
\bar{x}_{i-j+1}^{(21)} \\
\bar{x}_{i-j+1}^{(22)} \\
\vdots \\
\bar{x}_{i-j+1}^{\left(2, n_{2}\right)}
\end{array}\right]\right), i \in Z_{+}} \\
& =\left[\begin{array}{c}
\bar{x}_{i}^{(21)} \\
\bar{x}_{i}^{(22)} \\
\vdots \\
\bar{x}_{i}^{\left(2, n_{2}\right)}
\end{array}\right]+\left[\begin{array}{c}
B_{21} \\
B_{22} \\
\vdots \\
B_{2, n_{2}}
\end{array}\right] u_{i}
\end{aligned}
$$

From (6.10) we have:

$$
\begin{aligned}
& \bar{x}_{i}^{(21)}=-B_{21} u_{i} \\
& \bar{x}_{i}^{(22)}=\sum_{j=0}^{i+1}(-1)^{j}\left(\begin{array}{l}
\alpha \\
j
\end{array}\right) \bar{x}_{i-j+1}^{(21)}-B_{22} u_{i} \\
& =-\sum_{j=0}^{i+1}(-1)^{j}\left(\begin{array}{l}
\alpha \\
j
\end{array}\right) B_{21} u_{i-j+1}-B_{22} u_{i} \\
& \bar{x}_{i}^{(23)}=\sum_{j=0}^{i+1}(-1)^{j}\left(\begin{array}{l}
\alpha \\
j
\end{array}\right) \bar{x}_{i-j+1}^{(22)}-B_{23} u_{i} \\
& =-\sum_{j=0}^{i+1}(-1)^{j}\left(\begin{array}{c}
\alpha \\
j
\end{array}\right) \sum_{k=0}^{i-j+2}(-1)^{k}\left(\begin{array}{l}
\alpha \\
k
\end{array}\right) B_{21} u_{i-j-k+2} \\
& -\sum_{j=0}^{i+1}(-1)^{j}\left(\begin{array}{c}
\alpha \\
j
\end{array}\right) B_{22} u_{i-j+1}-B_{23} u_{i} \\
& \vdots \quad \bar{x}_{i}^{\left(2, n_{2}\right)}=\sum_{j=0}^{i+1}(-1)^{j}\left(\begin{array}{c}
\alpha \\
j
\end{array}\right) \bar{x}_{i-j+1}^{\left(2, n_{2}-1\right)}-B_{2, n_{2}} u_{i} \\
& \text { If } N=0 \text { then from (6.6) we have: }
\end{aligned}
$$$$
\bar{x}_{i}^{(2)}=-B_{2} u_{i}, i \in Z_{+}
$$

This approach can be easily extended for :

$$
N=\text { blockdiag }\left[\begin{array}{llll}
N_{1} & N_{2} & \ldots & N_{h}
\end{array}\right]
$$

where: $N_{k} \in \Re^{n_{k}}$ has the form (6.9) and $\sum_{i=1}^{h} n_{k}=n_{2}$. If the matrix $N$ has the form:

$$
N=\left[\begin{array}{ccccc}
0 & 1 & 0 & \ldots & 0 \\
0 & 0 & 1 & \ldots & 0 \\
\vdots & \vdots & \vdots & \ddots & \vdots \\
0 & 0 & 0 & \ldots & 1 \\
0 & 0 & 0 & \ldots & 0
\end{array}\right] \in \Re^{n_{2}}
$$

the considerations are similar (dual).

Note that the matrices (6.9) and (6.9') are related by:

$N=S \bar{N} S$ where $S=\left[\begin{array}{ccccc}0 & 0 & \ldots & 0 & 1 \\ 0 & 0 & \ldots & 1 & 0 \\ \vdots & \vdots & \ldots & \vdots & \vdots \\ 1 & 0 & \ldots & 0 & 0\end{array}\right]$.

Knowing $\bar{x}_{i}^{(1)}$ and $\bar{x}_{i}^{(2)}$ we can find the desired solution of Eq. (5.1) from (6.1):

$$
x_{i}=Q\left[\begin{array}{c}
\bar{x}_{i}^{(1)} \\
\bar{x}_{i}^{(2)}
\end{array}\right], i \in Z_{+}
$$

\section{EXAMPLES OF SINGULAR FRACTIONAL DISCRETE-TIME SYSTEMS}

Example 7.1. Find the solution $x_{i}$ of the singular fractional linear system (5.1) with the matrices:

$$
E=\left[\begin{array}{ccc}
-1 & -1 & -1 \\
2 & 4 & 2 \\
1 & 4 & 1
\end{array}\right], \quad A=\left[\begin{array}{lll}
0.8 & 1.7 & 2.8 \\
0.4 & 0.8 & 1.4 \\
2.2 & 4.6 & 2.2
\end{array}\right], \quad B=\left[\begin{array}{c}
1 \\
0 \\
-1
\end{array}\right]
$$

for $\alpha=0.5, u_{i}=u, i \in Z_{+}$and $x_{0}=\left[\begin{array}{lll}1 & 2 & -1\end{array}\right]^{T}(T$ denotes the transpose).

It is easy to check that the matrices (7.1) satisfy the assumptions (5.4). In this case the matrices $P$ and $Q$ have the forms:

$$
P=\frac{1}{11}\left[\begin{array}{ccc}
1 & -2 & 5 \\
-2 & 4 & 1 \\
4 & 3 & -2
\end{array}\right], \quad Q=\left[\begin{array}{ccc}
-2 & 1 & -1 \\
1 & 0 & 0 \\
0 & 0 & 1
\end{array}\right]
$$

and:

$$
\begin{aligned}
& {\left[\begin{array}{cc}
I_{n_{1}} & 0 \\
0 & N
\end{array}\right]=P E Q=\left[\begin{array}{lll}
1 & 0 & 0 \\
0 & 1 & 0 \\
0 & 0 & 0
\end{array}\right],} \\
& {\left[\begin{array}{cc}
A_{1} & 0 \\
0 & I_{n_{2}}
\end{array}\right]=P A Q=\left[\begin{array}{ccc}
0.1 & 1 & 0 \\
0 & 0.2 & 0 \\
0 & 0 & 1
\end{array}\right],}
\end{aligned}
$$

$$
P B=\left[\begin{array}{l}
B_{1} \\
B_{2}
\end{array}\right]=\frac{1}{11}\left[\begin{array}{c}
-4 \\
-3 \\
6
\end{array}\right], \quad A_{1 \alpha}=A_{1}+I_{n_{1}} \alpha=\left[\begin{array}{cc}
0.6 & 1 \\
0 & 0.7
\end{array}\right],
$$

$\left(n_{1}=2, n_{2}=1\right)$

The equations (6.5) and (6.6) have the forms:

$$
\bar{x}_{i+1}^{(1)}=\left[\begin{array}{cc}
0.6 & 1 \\
0 & 0.7
\end{array}\right] \bar{x}_{i}^{(1)}+\sum_{k=2}^{i+1}(-1)^{k-1}\left(\begin{array}{c}
0.5 \\
k
\end{array}\right) \bar{x}_{i-k+1}^{(1)}-\frac{1}{11}\left[\begin{array}{l}
4 \\
3
\end{array}\right] u_{i}, i \in Z_{+}
$$

and: 
$\bar{x}_{i}^{(2)}=-B_{2} u_{i}=-\frac{6}{11} u_{i}, i \in Z_{+}$

The solution $\bar{x}_{i}^{(1)}$ of the equation (7.4) has the form:

$\bar{x}_{i}^{(1)}=\Phi_{i} \bar{x}_{0}^{(1)}+\sum_{k=0}^{i-1} \Phi_{i-k-1} B_{1} u_{k}, i \in Z_{+}$

where:

$\Phi_{0}=\left[\begin{array}{ll}1 & 0 \\ 0 & 1\end{array}\right], \quad \Phi_{1}=A_{1 \alpha}=\left[\begin{array}{cc}0.6 & 1 \\ 0 & 0.7\end{array}\right]$

$\Phi_{2}=A_{1 \alpha}{ }^{2}-I_{n_{1}} \frac{\alpha(\alpha-1)}{2 !}=\left[\begin{array}{cc}0.485 & 1.300 \\ 0 & 0.615\end{array}\right], \cdots$

and:

$\bar{x}_{0}=Q^{-1} x_{0}=\left[\begin{array}{ccc}0 & 1 & 0 \\ 1 & 2 & 1 \\ 0 & 0 & 1\end{array}\right]\left[\begin{array}{c}1 \\ 2 \\ -1\end{array}\right]=\left[\begin{array}{c}2 \\ 4 \\ -1\end{array}\right]$,

$\bar{x}_{0}^{(1)}=\left[\begin{array}{l}2 \\ 4\end{array}\right], \quad \bar{x}_{0}^{(2)}=[-1]$

The desired solution of the singular fractional system with (7.1) is given by:

$$
x_{i}=Q \bar{x}_{i}=\left[\begin{array}{ccc}
-2 & 1 & -1 \\
1 & 0 & 0 \\
0 & 0 & 1
\end{array}\right]\left[\begin{array}{c}
\bar{x}_{i}^{(1)} \\
\bar{x}_{i}^{(2)}
\end{array}\right]
$$

where $\bar{x}_{i}^{(1)}$ and $\bar{x}_{i}^{(2)}$ are determined by (6.7) and (7.5), respectively.

Example 7.2. Find the solution $x_{i}$ of the singular fractional linear system (5.1) with the matrices:

$E=\left[\begin{array}{ccc}1 & 0 & 0 \\ 0 & 1 & -1 \\ 1 & -1 & 1\end{array}\right], \quad A=\left[\begin{array}{ccc}0.2 & 2 & -2 \\ 2 & 1 & 0 \\ -1.8 & 0 & -1\end{array}\right], \quad B=\left[\begin{array}{cc}1 & 2 \\ -1 & 2 \\ 2 & -1\end{array}\right]$

for $\alpha=0.8$, arbitrary $u_{i,} i \in Z_{+}$and $x_{0}=\left[\begin{array}{lll}1 & 1 & 1\end{array}\right]^{T}$.

It is easy to check that the matrices (7.10) satisfy the assumptions (5.4). In this case the matrices $P$ and $Q$ have the forms:

$$
P=\left[\begin{array}{ccc}
-1 & 2 & 2 \\
1 & -1 & -1 \\
-1 & 2 & 1
\end{array}\right], Q=\left[\begin{array}{ccc}
1 & 0 & 0 \\
-2 & 1 & 1 \\
-2 & 0 & 1
\end{array}\right]
$$

and:

$$
\begin{aligned}
& {\left[\begin{array}{cc}
I_{n_{1}} & 0 \\
0 & N
\end{array}\right]=P E Q=\left[\begin{array}{lll}
1 & 0 & 0 \\
0 & 0 & 0 \\
0 & 1 & 0
\end{array}\right],} \\
& {\left[\begin{array}{cc}
A_{1} & 0 \\
0 & I_{n_{2}}
\end{array}\right]=P A Q=\left[\begin{array}{ccc}
0.2 & 0 & 0 \\
0 & 1 & 0 \\
0 & 0 & 1
\end{array}\right],} \\
& P B=\left[\begin{array}{l}
B_{1} \\
B_{2}
\end{array}\right]=\left[\begin{array}{cc}
1 & 0 \\
0 & 1 \\
-1 & 1
\end{array}\right], A_{1 \alpha}=A_{1}+I_{n_{1}} \alpha=[1], \\
& \left(n_{1}=1, n_{2}=2\right)
\end{aligned}
$$

In this case the equations (6.5) and (6.6) have the forms:

$$
\begin{aligned}
& \bar{x}_{i+1}^{(1)}=\bar{x}_{i}^{(1)}+\sum_{k=2}^{i+1}(-1)^{k-1}\left(\begin{array}{c}
0.8 \\
k
\end{array}\right) \bar{x}_{i-k+1}^{(1)}+\left[\begin{array}{ll}
1 & 0
\end{array}\right] u_{i}, i \in Z_{+} \quad \text { (7.13) } \\
& {\left[\begin{array}{ll}
0 & 0 \\
1 & 0
\end{array}\right]\left(\sum_{j=0}^{i+1}(-1)^{j}\left(\begin{array}{c}
0.8 \\
j
\end{array}\right)\left[\begin{array}{l}
\bar{x}_{i-j+1}^{(21)} \\
\bar{x}_{i-j+1}^{(22)}
\end{array}\right]\right)=\left[\begin{array}{c}
\bar{x}_{i}^{(21)} \\
\bar{x}_{i}^{(22)}
\end{array}\right]+\left[\begin{array}{cc}
0 & 1 \\
-1 & 1
\end{array}\right] u_{i}, i \in Z_{+}}
\end{aligned}
$$

and:

$$
\bar{x}_{0}=Q^{-1} x_{0}=\left[\begin{array}{ccc}
1 & 0 & 0 \\
0 & 1 & -1 \\
2 & 0 & 1
\end{array}\right]\left[\begin{array}{l}
1 \\
1 \\
1
\end{array}\right]=\left[\begin{array}{l}
1 \\
0 \\
3
\end{array}\right], \quad \bar{x}_{0}^{(1)}=[1], \quad \bar{x}_{0}^{(2)}=\left[\begin{array}{l}
0 \\
3
\end{array}\right]
$$

The solution $\bar{x}_{i}^{(1)}$ of Eq. (7.13) with $\bar{x}_{0}^{(1)}=1$ can be easily found using (6.7) and (6.8).

From (7.14) we have:

$$
\begin{aligned}
& \bar{x}_{i}^{(21)}=\left[\begin{array}{ll}
0 & -1
\end{array}\right] u_{i}, \quad i \in Z_{+} \\
& \bar{x}_{i}^{(22)}=\sum_{j=0}^{i+1}(-1)^{j}\left(\begin{array}{c}
0.8 \\
j
\end{array}\right)\left[\begin{array}{ll}
0 & -1
\end{array}\right] u_{i-j+1}+\left[\begin{array}{ll}
1 & -1
\end{array}\right] u_{i}, \quad i \in Z_{+}
\end{aligned}
$$

The desired solution of the singular fractional system with (7.10) is given by:

$$
x_{i}=Q \bar{x}_{i}=\left[\begin{array}{ccc}
1 & 0 & 0 \\
-2 & 1 & 1 \\
-2 & 0 & 1
\end{array}\right]\left[\begin{array}{c}
\bar{x}_{i}^{(1)} \\
\bar{x}_{i}^{(21)} \\
\bar{x}_{i}^{(22)}
\end{array}\right]
$$

where: $\bar{x}_{i}^{(1)}, \bar{x}_{i}^{(21)}$ and $\bar{x}_{i}^{(22)}$ are determined by (7.13) and (7.16), respectively.

\section{CONCLUDING REMARKS}

The singular fractional linear systems and electrical circuits have been introduced. Using the Caputo definition of the fractional derivative, the Weierstrass regular pencil decomposition and the Laplace transform the solution to the state equation of singular fractional linear system has been derived (Theorem 3.1). Singular 
fractional linear electrical circuits have been analyzed. It has been shown that every electrical circuit is a singular fractional system if it contains at least one mesh consisting of branches with only ideal supercondensators and voltage sources or at least one node with branches with supercoils (Theorem 4.1). The singular fractional linear discrete-time systems have been introduced. Using the Weierstrass regular pencil decomposition the solution to the state equation of singular fractional linear discrete-time system has been derived. The method of finding of the solution to the singular fractional systems has been illustrated by two examples. The considerations have been illustrated by singular linear electrical circuits. Those considerations can be extended for singular fractional linear systems with singular pencils. Open problem are extension of these considerations for positive singular fractional linear systems and for singular positive linear systems with different fractional order. The linear systems with different fractional orders are described by the equation (Kaczorek, 2007a).

$$
\begin{aligned}
& {\left[\begin{array}{l}
\frac{d^{\alpha} x_{1}}{d t^{\alpha}} \\
\frac{d^{\beta} x_{2}}{d t^{\beta}}
\end{array}\right]=\left[\begin{array}{ll}
A_{11} & A_{12} \\
A_{21} & A_{22}
\end{array}\right]\left[\begin{array}{l}
x_{1} \\
x_{2}
\end{array}\right]+\left[\begin{array}{l}
B_{1} \\
B_{2}
\end{array}\right] u,} \\
& p-1<\alpha<p ; \quad q-1<\beta<q ; \quad p, q \in N
\end{aligned}
$$

where: $x_{1} \in \mathfrak{R}^{n_{1}}, x_{2} \in \mathfrak{R}^{n_{2}}$ are the state vectors and $A_{i j} \in$ $\mathfrak{R}^{n_{i} \times n_{j}}, \quad B_{i} \in \mathfrak{R}^{n_{i} \times m}, \quad i, j=1,2$ and $u \in \mathfrak{R}^{m}$ is the input vector. Initial conditions for (8.1) have the form $x_{1}(0)=x_{10}$ and $x_{2}(0)=x_{20}$.

\section{REFERENCES}

1. Benvenuti L., Farina L. (2004), A tutorial on the positive realization problem, IEEE Trans. Autom. Control., Vol. 49, No. 5, 651-664.

2. Dail L. (1989), Singular control systems, Lectures Notes in Control and Information Sciences, Springer-Verlag, Berlin.

3. Dodig M. Stosic M. (2009), Singular systems state feedbacks problems, Linear Algebra and its Applications, Vol. 431, No. 8, 1267-1292.

4. Fahmy M.H, O'Reill J. (1989), Matrix pencil of closed-loop descriptor systems: infinite-eigenvalues assignment, Int. J. Control., Vol. 49, No. 4, 1421-1431.

5. Gantmacher F. R. (1960), The theory of Matrices, Chelsea Publishing Co., New York.

6. Kaczorek T. (1992), Linear control systems, Vol. 1, Research Studies Press J. Wiley, New York.

7. Kaczorek T. (2004), Infinite eigenvalue assignment by outputfeedbacks for singular systems, Int. J. Appl. Math. Comput. Sci., Vol. 14, No. 1, 19-23.

8. Kaczorek T. (2007a), Polynomial and rational matrices. Applications in dynamical systems theory, Springer-Verlag, London.

9. Kaczorek T. (2007b), Realization problem for singular positive continuous-time systems with delays, Control and Cybernetics, Vol. 36, No. 1, 47-57.

10. Kaczorek T. (2008), Fractional positive continuous-time linear systems and their reachability, Int. J. Appl. Math. Comput. Sci., Vol. 18, No. 2, 223-228.

11. Kaczorek T. (2010a), Analysis of fractional electrical circuits in transient states, VII Konferencja Naukowo-Techniczna : Logistyka - systemy transportowe - bezpieczeństwo w transporcie, Szczyrk.

12. Kaczorek T. (2010b), Positive linear systems with different fractional orders, Bull. Pol. Ac. Sci. Techn., Vol. 58, No. 3, 453-458.

13. Kaczorek T. (2011) Selected Problems in Fractional Systems Theory, Springer-Verlag.

14. Kucera V. Zagalak P. (1988), Fundamental theorem of state feedback for singular systems, Automatica, Vol. 24, No. 5, 653-658.

15. Podlubny I. (1999), Fractional differential equations, Academic Press, New York.

16. Van Dooren P. (1979), The computation of Kronecker's canonical form of a singular pencil, Linear Algebra and Its Applications, Vol. 27, 103-140.

This work was supported by National Science Centre in Poland under work no. NN514 193933. 